\title{
Die Erneuerung der Schweizerischen Bibliothekenstatistik
}

\begin{abstract}
Der Artikel beschreibt die jahrhundertealte Geschichte der Schweizerischen Bibliothekenstatistik (nicht: Bibliotheksstatistik), deren komplette Neugestaltung zu Beginn des 21. Jahrhunderts und die angekündigte Reduktion auf wenige aggregierte Werte. Insbesondere die Aufnahme von Kennzahlen zur Sammlung und Nutzung von elektronischen Medien stellte für alle Beteiligten eine grosse Herausforderung dar.
\end{abstract}

\section{Blick in die Geschichte}

In den 1990er Jahren wurde die 1960 eingeführte Schweizerische Bibliothekenstatistik immer stärker als veraltet empfunden. Dies vor allem aus zwei Gründen: Erstens wurde nur eine enge Auswahl von 47 Bibliotheken befragt. Zweitens fokussierten die erhobenen Kennzahlen zu stark auf Finanzen, Bestand und Personal, waren nicht Output-orientiert und negierten neue Entwicklungen wie elektronische Medien. Im Auftrag des Verbands der Bibliotheken und Bibliothekarinnen/Bibliothekare der Schweiz (BBS) konzipierte eine Arbeitsgruppe das neue Kennzahlenraster und schlug eine neue Auswahl von Bibliotheken in enger Zusammenarbeit mit dem Bundesamt für Statistik (BFS) vor. Nach Überwindung von Anfangsschwierigkeiten wurde die neue Statistik breit akzeptiert. Einzig das dynamische Feld der elektronischen Medien bleibt ein Sorgenkind. Nach einem moderaten Ausbau der Kennzahlen auf rund 80 gehen die jüngsten Vorschläge des BFS in Richtung einer massiven Reduktion der Anzahl erfasster Werte.

Die Schweizerische Bibliothekenstatistik hat eine lange Geschichte: Nach zwei Vollerhebungen bei rund 6'000 Bibliotheken in der Schweiz in den Jahren 1868 und 1911 wurden für wenige ausgewählte Bibliotheken ab 1927 jährlich Zahlen gesammelt. Ab 1960 bis 2002 konnte die Statistik vom Eidgenössischen Statistischen Amt, später Bundesamt für Statistik, alljährlich durchgeführt werden. Sie lieferte Datenreihen zu 63 Kennzahlen aus den Bereichen Einnahmen, Ausgaben, Personal, Bestand, Zuwachs und Benutzung, dies allerdings nur für eine enge Auswahl von 47 vom BFS ausgewählten Öffentlichen Bibliotheken. Die Bibliotheken wurden zu Vergleichszwecken in drei Kategorien eingeteilt:

Ә Open Access. () Beat A. Wartmann, publiziert von De Gruyter. (c) BY-NC-ND Dieses Werk ist lizenziert unter der Creative Commons Attribution-NonCommercial-NoDerivatives 4.0 Lizenz. https://doi.org/10.1515/9783110553796-034 
1. Die ,Wissenschaftlichen Bibliotheken“ umfassten die Schweizerische Landesbibliothek, elf Universitätsbibliotheken sowie die zwei Spezialbibliotheken Schweizerisches Wirtschaftsarchiv Basel (bis 1987, danach integriert in UB Basel) und Schweizerisches Sozialarchiv Zürich

2. Die Kategorie ,Volksbibliotheken', 1995 umbenannt in ,Allgemeine Öffentliche Bibliotheken', umfasste nur sieben bedeutende Bibliotheken der Städte Basel, Bern (Bibliomedia und Kornhausbibliotheken), Genf, Lausanne, Winterthur (Kreisbibliotheken) und Zürich (Pestalozzi Bibliothek Zürich, PBZ)

3. Die Kategorie ,Studien- und Bildungsbibliotheken' umfasste 18 Kantonsbibliotheken sowie die Stadtbibliotheken von Baden, Biel, La Chaux-deFonds, Olten, St. Gallen, Thun, Winterthur und Zofingen.

\section{Neukonzeption der Bibliothekenstatistik in den Nullerjahren}

Im ausgehenden 20. Jahrhundert machte sich in der Schweizerischen Bibliothekslandschaft ein Unbehagen über diese immer mehr als willkürlich empfundene Auswahl an Bibliotheken und Kennzahlen bemerkbar. So fehlten die Universität St. Gallen ebenso wie die neu entstandenen Fachhochschulen. Auch entsprachen die erhobenen Kennzahlen nicht mehr den angebotenen Dienstleistungen im Zeitalter der aufkommenden elektronischen Medien. Nach ausgiebigen Diskussionen beschloss der Vorstand des Verbands der Bibliotheken und Bibliothekarinnen/Bibliothekare der Schweiz am 19. Oktober 2000, eine Arbeitsgruppe zur Neubearbeitung der Schweizerischen Bibliothekenstatistik einzusetzen. Das Mandat des BBS für diese Arbeitsgruppe wurde wie folgt umschrieben: „Die Arbeitsgruppe Statistik erarbeitet die Grundlagen für eine Schweizerische Bibliotheksstatistik, die alle Bibliothekstypen einschliesst. Sie orientiert sich dabei an europäischen Standards. Sie sucht von Beginn weg die Zusammenarbeit mit dem Bundesamt für Statistik. Sie klärt ab, welche politischen Vorstösse notwendig sind, damit das Bundesamt für Statistik auch die neue Bibliotheksstatistik betreut."1 Die Arbeitsgruppe konstituierte sich auf ihrer ersten Sitzung am 15. Februar 2001 im Sekretariat des BBS mit folgenden Vertretern: Dr. Wilfried Lochbühler (ZHB Luzern, Präsident), Hansueli Locher (SLB), Alexis Rivier (BPU Genf), Dr. Hermann Romer (Stadtbibliothek Winterthur) und Dr. Beat Wartmann (ZB

1 Vorstandsbeschluss des BBS vom 19.10.2000. 
Zürich). Am 19. März 2001 hiess der Direktor des Bundesamtes für Statistik Dr. Carlo Malaguerra die Initiative des BBS gut und entsandte einen Vertreter in die Arbeitsgruppe.

Die AG Statistik begann ihre Arbeit mit dem Ziel, einen aktuellen Leistungsnachweis mit aggregierten Kennzahlen zu erbringen, der

- als Führungs- und Managementinstrument dienen,

- die modifizierten Kennzahlen den veränderten Anforderungen anpassen,

- die langfristige Leistungs- und Betriebsentwicklung weiterhin dokumentieren,

- $\quad$ einen Überblick auf eine repräsentative Auswahl von etwa 150 Bibliotheken eine Vorgabe des BFS - liefern sowie

- die Kompatibilität mit internationalen Normen sicherstellen sollte.

Für das Kennzahlenraster diente als Referenzgrundlage das Benchmarking-Modell der Studien- und Bildungsbibliotheken der Schweiz. Dieses musste aber erheblich modifiziert und teilweise erweitert werden. Um die Anzahl der erhobenen Kennzahlen nicht massiv zu erhöhen, wurden die Angaben zu Bestand, Zuwachs und Personal gestrafft, dafür Zielgruppen und Kunden erfasst, eine stärkere Output-Orientierung angestrebt sowie die elektronischen Medien neu berücksichtigt.

Als fast noch komplexer als das Kennzahlenraster erwies sich die Auswahl der teilnehmenden Bibliotheken. In die Kategorie ,Bibliotheken mit nationalem Auftrag' wurden neu die Landesphonothek und die Cinémathèque Suisse aufgenommen, alle 14 Universitätsbibliotheken sollten neu vertreten sein, aber auch die zahlreichen Instituts- und Seminarbibliotheken als universitäre Netze im Sinne einer administrativen Einheit. Für die Auswahl der Öffentlichen Bibliotheken standen 70 Plätze zur Verfügung, die aufgrund eines Zentren-Modells des BFS (politische Gemeinden mit Zentrumsfunktion) und aufgrund der Rückmeldung von regionalen Bibliotheksexperten zugewiesen wurden. Dabei fanden auch Mindestkriterien wie Bestandsgrösse, Öffnungszeiten und Professionalität Anwendung. Für die sich dynamisch entwickelnden Fachhochschulen wurde eine Aggregierung der rund 80 Institutionen $\mathrm{zu}$ den sieben FH-Regionen der Schweiz angestrebt. Für die Mittelschul- und Berufsschulbibliotheken wurden auch Mindeststandards definiert. Aus 117 befragten Spezialbibliotheken wurden schliesslich mittels eines Medianwerts von acht Kennzahlen zu Personal, Bestand und Zuwachs diejenigen 44 Bibliotheken in die Statistik aufgenommen, welche diesen überschritten.

Die umfangreiche Vernehmlassung der geplanten neuen Statistik wurde im März 2002 durch das BBS-Sekretariat durchgeführt. Die Fachwelt wurde durch zwei Artikel gleichen Inhalts in $S D B / B D S$-News und arbido (Lochbühler 
2002) $)^{2}$ zur Vernehmlassung eingeladen. Die 150 ausgewählten Referenzbibliotheken wurden direkt zur Vernehmlassung aufgefordert, ansonsten wurden die Dachorganisationen, Interessengruppen und Erziehungsdirektionen angeschrieben.

Ab September 2002 hat die AG Statistik die Ergebnisse der Vernehmlassung gesichtet und die Probebefragung 2003 vorbereitet. Die 101 eingegangenen detaillierten Stellungnahmen waren insgesamt positiv. Begrüsst wurden die neue strategische Ausrichtung des aktualisierten Kennzahlenrasters, die stärkere OutputOrientierung wie auch der Einbezug neuer Bibliothekskategorien. Die Bereitschaft der Erziehungsdirektionen, die Daten ihrer Mittel- und Berufsschulbibliotheken zusammenzustellen, war verhalten bis negativ. Eher gering fiel das Interesse der Spezialbibliotheken aus. Bezüglich der Kennzahlen kamen viele Verbesserungsvorschläge, welche eine Überarbeitung des schwierigen Komplexes ,Elektronische Medien' nötig machte. Es zeigte sich, dass zahlreiche Bibliotheken nicht in der Lage waren, für die Nutzung elektronischer Medien vernünftige Angaben zu liefern (Lochbühler 2003) ${ }^{3}$.

Anfang Mai 2003 startete die Probebefragung (Erhebungsjahr 2002) auf dem Server des BBS bei den 140 schliesslich ausgewählten Referenzbibliotheken. Dabei ging es hauptsächlich darum, alle Bibliotheken (auch die ,Etablierten') an die neuen Kennzahlen zu gewöhnen, mussten doch teilweise gänzlich neue Erhebungsverfahren entwickelt und getestet werden. Da die Daten des Jahres 2002 vorgängig zur Probebefragung noch auf dem bisherigen Weg erhoben worden waren und auch publiziert wurden ${ }^{4}$, konnte auf eine Publikation der Daten aus der Probebefragung verzichtet werden. Die Befragung erfolgte mittels eines passwortgeschützten Fragebogens mit Eingabe in ein Excel-Formular. Erstmals wurden auch sechs Uni-Netze mit 283 Teilbibliotheken und sieben FH-Netze mit 88 Teilbibliotheken erfasst. Die Probebefragung erzielte bei den Bibliothekstypen unterschiedliche Resonanz: Während Uni-Netze (100\%) und Universitätsbibliotheken (79\%) gut repräsentiert waren, waren die Rückläufe von Öffentlichen Bibliotheken (60\%), Spezialbibliotheken (48\%) und FH-Bibliotheken (29\%) ungenügend. Einmal mehr zeigten sich gravierende Erhebungsprobleme im Bereich der elektronischen Medien, weil die Nutzungszahlen auf ganz unterschiedlichen Grundlagen basierten und teilweise nur aggregiert für ein ganzes Netz von Biblio-

2 Wilfried Lochbühler: Zur neuen Bibliotheksstatistik der Schweiz. In: arbido 3/2002, S. 18-20; wieder in SDB/BDS-News 63 (2002), S. 29-32.

3 Wilfried Lochbühler: Zur neuen Bibliotheksstatistik der Schweiz. Zwischenbericht 2003 der Arbeitsgruppe Statistik des BBS. In: arbido 4/2003, S. 21-22.

4 Bundesamt für Statistik/Schweizerische Bibliotheken. Statistische Übersichten 2002. Bern 2003. 
theken zur Verfügung standen. Aufgrund der eingegangenen Rückmeldungen konnte die Arbeitsgruppe eine ganze Reihe von Präzisierungen der Definitionen und Ergänzungen am Fragebogen vornehmen (Lochbühler 2004) ${ }^{5}$.

In ihrer Sitzung am 28. April 2004 hat die AG Statistik alle noch offenen Fragen der Darstellung des Fragebogens geklärt. Somit konnte das BFS Ende April die neue Bibliothekenstatistik definitiv einführen. Damit war der Auftrag des BBS an die Arbeitsgruppe erfüllt und diese aufgelöst. Weil das Bundesamt für Statistik jedoch eine weitere Begleitung durch ein Fachgremium wünschte, verlängerte der Vorstand des BBS in seiner Sitzung vom 10. Februar 2004 das Mandat um weitere drei Jahre bis 2006.

Die Resultate der ersten Haupterhebung nach neuem Muster liessen sich sehen: Bibliotheken mit nationalem Auftrag und Universitätsbibliotheken antworteten zu 100\%. Die Koordination der Erhebungen bei den Uni-Netzen erforderte viel Aufwand für Motivation und Kontrolle, doch machten auch alle Netze mit unterschiedlichen Anteilen der Teilbibliotheken mit. Leider blieben die Probleme bei der Nutzung elektronischer Medien nach wie vor bestehen. Bei den Öffentlichen Bibliotheken lag der Rücklauf bei guten 81\%, von den Fachhochschulen konnten nur die Zentralschweiz und Zürich erfasst werden. Bei den Spezialbibliotheken war zwar der Rücklauf mit 75\% befriedigend, aber einige methodische Fragen blieben ungelöst. Die Ergebnisse der Erhebung konnten dank der Einwilligung aller Direktionen auf dem Portal des BFS als Rohdaten publiziert werden, so dass Vergleiche zwischen den Bibliotheken möglich wurden (Lochbühler 2005) ${ }^{6}$.

2005 begann die Arbeitsgruppe über die Auswahl der Öffentlichen Bibliotheken nachzudenken. Das bisherige Regionenmodell mit Expertenauswahl wurde von den Statistikfachleuten des BFS nun in Frage gestellt und ein neues ,statistisches Universum' angestrebt, nämlich sämtliche Stadtbibliotheken. Als ,Stadt‘ definiert das BFS jede Gemeinde mit mehr als 10’000 Einwohnern, damals waren dies etwa 120. Die AG Statistik entwickelte einen vereinfachten Fragebogen mit nur 35 Kennzahlen für Städte mit weniger als 20’000 Einwohnern, weil absehbar war, dass diese relativ kleinen Bibliotheken nicht in der Lage wären, alle 72 Kennzahlen zu beantworten. Die Probebefragung bei 73 angeschriebenen Stadtbibliotheken war mit einem Rücklauf von nur 30\% unbefriedigend. Das BBS-Sekretariat wurde um Unterstützung gebeten, um Überzeugungsarbeit zu leisten.

5 Wilfried Lochbühler: Zur neuen Bibliotheksstatistik der Schweiz. Bilanz der Probebefragung 2003 und definitive Einführung 2004. In: arbido 5/2004, S. 60-62.

6 Wilfried Lochbühler: Zur neuen Bibliotheksstatistik der Schweiz. Bilanz der ersten Hauptbefragung durch das BFS 2004. In: arbido 7+8/2005, S. 16-18. 
Im Herbst 2005 machte das BFS eine Umfrage bei den teilnehmenden Bibliotheken bezüglich Informationsgehalt, Präsentation, konkretem Nutzen versus Aufwand der neuen Bibliothekenstatistik. Die Antworten variierten stark je nach Bibliothekstyp. Die Koordinationsstellen der Uni-Netze äusserten sich negativ bezüglich des zu leistenden Aufwands. Inhaltliche Kritik an der Statistik kam nur von den Koordinationsstellen der Kantone Bern und Zürich, ansonsten wurden die erhobenen Kennzahlen nicht hinterfragt.

Die zweite Haupterhebung 2005 (Daten 2004) erbrachte weiterhin 100\%ige Rücklaufquoten für Bibliotheken mit nationalem Auftrag, Universitätsbibliotheken und Bibliotheksnetze der Universitäten, aber ungenügende bei Fachhochschulen und Öffentlichen Bibliotheken. Die dritte Haupterhebung 2006 (Daten 2005) wurde erstmals auch bei 128 Öffentlichen Bibliotheken, darunter 68 neu aufgenommenen, mit einer Rücklaufquote von 78\% durchgeführt. Aufgrund fehlender Ressourcen und methodischer Mängel entschied das BFS Mitte 2006, die Kategorie ,Spezialbibliotheken“ nicht mehr zu erheben. Damit fiel das Schweizerische Sozialarchiv, welches seit 1960 in der alten Statistik erfasst worden war, jetzt aus der Statistik.

2006 lag der Schwerpunkt der Arbeitsgruppe Statistik auf der Vorbereitung der Online-Datenerhebung via eSurvey auf dem Server des BFS. Dabei wurde die Erfassung der Zahlen zum Bibliotheken-Benchmarking gleich mitgedacht. Dieses Benchmarking wurde 1998 als Projekt der IG Studien- und Bildungsbibliotheken des BBS initiiert und seit 2002 vom Institut für Verwaltungs-Management der Zürcher Fachhochschule Winterthur (IVM) organisiert (Frei 2005) ${ }^{7}$. Es umfasste 200621 Bibliotheken in den Vergleichskreisen ,Wissenschaftliche Universitätsbibliotheken“, ,Studien- und Bildungsbibliotheken', ,Regional- und Stadtbibliotheken' sowie ,Stadtbibliotheken mit Filialnetz'. Im Vordergrund stand der direkte Leistungsvergleich mittels Leistungsindikatoren (Verhältniskennzahlen) und umfasste auch vertrauliche interne Betriebszahlen, weshalb nur die Teilnehmerbibliotheken Zugriff auf die Zahlen erhielten und eine Veröffentlichung nicht in Frage kam (Romer 2005) ${ }^{8}$. Dank einer Vereinbarung zwischen BFS und IVM konnte ab 2007 die Datenerhebung in einem für das Benchmarking erweiterten Fragebogen gleichzeitig mit der Bibliothekenstatistik auf dem eSurvey-Tool erfolgen. Der BBS-Vorstand verlängerte das Mandat der AG Statistik nicht um weitere drei Jahre, sondern wandelte die AG zum 01. Januar 2007 in eine permanente Arbeitsgruppe um.

7 Rebecca Frei: Bibliotheken-Benchmarking. In: arbido 11/2005, S. 5-6.

8 Hermann Romer: Herausforderung Benchmarking. Das schweizerische Bibliotheken-Benchmarking als Führungsinstrument. In: arbido 11/2005, S. 7-8. 
2007 wandte sich das BFS an die kantonalen Erziehungsdirektionen bzw., wo vorhanden, an kantonale Bibliotheksbeauftragte, um eine Vereinheitlichung der Daten zu den Gemeinde- und Schulbibliotheken anzudenken. Im Herbst 2007 fand eine erste Anhörung von elf Kantonen statt, 2008 konnte mit acht Kantonen (AG, AR, BE, LU, SG, SO, VS, ZH) eine Vereinbarung zur entgeltlichen Zusammenarbeit für die Jahre 2009 bis 2011 abgeschlossen werden. Eine Pilotbefragung 2008 diente dazu, die Datenerhebung zu organisieren und das Verfahren zu testen. 2011/2012 konnten mit GR, TG und OW drei weitere Kantone ins Boot geholt werden. 2012 publizierte das BFS die Daten von insgesamt 507 Gemeindebibliotheken und kombinierten Gemeinde- und Schulbibliotheken, womit eine gewichtige Erweiterung der Schweizerischen Bibliothekenstatistik erreicht werden konnte. Ab 2014 (Daten 2013) wurden schliesslich auch die Bibliotheken der Pädagogischen Hochschulen erfasst, womit eine wichtige Lücke im Bereich der Hochschulbibliotheken geschlossen werden konnte.

\section{Integration der elektronischen Medien und andere neue Entwicklungen}

Zehn Jahre nach der Einführung der neuen Schweizerischen Bibliothekenstatistik war 2014 die Zeit gekommen, die erfassten Kennzahlen kritisch zu hinterfragen und neue Entwicklungen, vor allem im Bereich der elektronischen Medien, nachzuvollziehen. Bereits 2013 hatte die Arbeitsgruppe begonnen, den Fragebogen im Detail zu überarbeiten. Folgende Kennzahlen wurden neu vorgeschlagen:

- Handschriften (Bestand): Anzahl Laufmeter als Messgrösse für Nachlässe

- elektronische Zeitschriften (Bestand): lizenzierte Titel als Teilmenge des gesamten Angebots, welches auch Open Access umfasst

- Datenbanken, E-Books und digitale Einzeldokumente (Bestand): neu getrennt erfasst

- Datenbanken (Nutzung): gemäss dem neuen internationalen Standard Counter 4 werden neu aufgerufene Datensätze statt Zugriffe gezählt

- E-Books und Elektronische Zeitschriften (Nutzung): Neudownloads

- digitale AV-Medien (Nutzung): Neudownloads und Streaming

- Führungen, Kurse, Lehrveranstaltungen: Anzahl Anlässe erweitert um Kurse und Lehrveranstaltungen, neu ebenfalls Dauer in Stunden und Anzahl Teilnehmende

Im Frühjahr 2014 wurde bei 245 Bibliotheken eine Pilotumfrage zu den neuen Kennzahlen durchgeführt. Aufgrund der eingegangenen Reaktionen wurden die 
Definitionen angepasst. Auf die Erhebung der Nutzung sozialer Medien wurde verzichtet. 2015 war ein Übergangsjahr, in welchem die Bibliotheken angehalten waren, die Datenerhebung der neuen Kennzahlen zu organisieren, so dass 2016 die Haupterhebung mit dem erweiterten Kennzahlenraster erfolgen konnte. Alle diese Neuerungen änderten nichts an der Tatsache, dass die Nutzung elektronischer Medien teilweise schwierig zu erfassen ist. In Bibliotheksnetzen mit zentralem Einkauf können nur Zahlen für das gesamte Netz erhoben werden, so etwa in den Netzen der Fachhochschulen. In Netzen, wo zwei oder mehr Partner sich die Erwerbung teilen, aber die Nutzung gemeinsam organisieren, können die Zahlen nur zentral erhoben werden, allenfalls muss die Nutzung künstlich getrennt werden, etwa proportional nach Erwerbungsbudget für elektronische Medien.

Etwas überraschend für die Arbeitsgruppe kam am 23. August 2016 vom BFS ein Vorschlag zur völligen Neugestaltung und massiven Reduktion des Kennzahlenrasters von derzeit rund 80 Variablen. Das Bundesamt präzisierte den Revisionsbedarf am 01. Februar 2017 folgendermassen:

- Es sind nicht alle erfassten Variablen statistisch und gesellschaftspolitisch gleichermassen relevant. Die (zu) grosse Zahl der erfassten Variablen bringt es zudem mit sich, dass nicht alle Daten vollständig erfasst sind, was Probleme bei der Analyse schafft.

- Es wird nicht das gesamte Bibliotheken-Universum erfasst. Über den wichtigen Typus der Öffentlichen Bibliotheken gibt es für die gesamte Schweiz keine Zahlen, sondern derzeit nur für 13 Kantone.

- Das Publizieren von Individualdaten ist keine Aufgabe der Statistik. Statistik hat primär die Aufgabe, aus Individualdaten relevante aggregierte Daten zu bilden, zu analysieren und zu publizieren.

- Zu einem Problem könnte - angesichts der eingeführten Kosten-LeistungsRechnung des Bundes - die heutige Ausgestaltung der Finanzierung der Bibliothekenstatistik werden. Zurzeit besteht ein Ungleichgewicht zwischen ,Finanzierern' und ,Mitfahrern'?

Zur Erarbeitung des Detailkonzeptes will das BFS eine Begleitgruppe einsetzen, welche neben vier Vertretern des BFS auch externe Vertreter umfassen soll:

- Bundesamt für Kultur (BAK): 1 Vertreter

- Nationalbibliothek (NB): Patrice Landry

- Bibliothek Information Schweiz (BIS): Thomas Wieland

9 Gewisse Bibliothekskategorien müssen für die Teilnahme an der Statistik bezahlen, dies sind die ,Finanzierer'. 
- Universitätsbibliotheken: Marianne Ingold (neue Präsidentin der AG Statistik ab Mai 2017)

- Fachhochschulbibliotheken: Anne Pfeiffer (Berner FH)

- Öffentliche Bibliotheken: Thomas Wieland (Kantonsbibliothek St. Gallen)

- Forschung: Prof. Sebastian Mundt (Hochschule der Medien, Stuttgart)

- Benchmarking: Martina Flick Witzig (IVW)

Die Fokussierung des BFS auf ,Vollständigkeit‘ des Universums der Öffentlichen Bibliotheken ebenso wie die Vorstellungen des Bundesamts zur Finanzierung wird noch viel $\mathrm{zu}$ reden geben. Nicht zuletzt wird auch der Berufsverband BIS gefordert sein, wenn die Datenreihen der Individualzahlen einer Reihe bedeutender Bibliotheken weitergeführt werden sollen. 